\title{
AVALIAÇÃO DA RESPOSTA DO PLASMODIUM FALCIPARUM À CLOROQUINA, QUININO E MEFLOQUINA (1)
}

\author{
S.M. DI SANTI (2), V.L.F, CAMARgO NeVES (2), M. BOUlOS (2), A.P. DUTRA (2),
}

A.M.S.V. RAMOS (2), M. SANTOS (2) \& L.C.B. BARATA (2)

\begin{abstract}
RESUMO
Nosso estudo envolveu a análise de cepas de Plasmodium falciparum provenientes da Região Amazônica Brasileira, coletadas no Laboratório de Malária da SUCEN. Os estudos "in vitro" foram efetuados com a cloroquina (46 ensaios), quinino (42 ensaios) e mefloquina (51 ensaios). Os resultados mostraram resistência de $100 \%$ em relaçào à cloroquina, $2,4 \%$ ao quinino e $31,4 \%$ à mefloquina, na análise "in vitro". Sete pacientes foram tratados com quinino isolado e nove com a associação mefloquina + pirimetamina-sulfadoxina, não mostrando correlação com os testes "in vitro".
\end{abstract}

UNitermos: Terapèutica da malaria; Microtestes de sensibilidade ao Plasmodium falciparum.

\section{INTRODUÇÃO}

A resistência do Plasmodium falciparum a diversos antimaláricos, isto é, sua capacidade de multiplicar-se ou sobreviver em presença de determinada concentração de uma droga que normalmente é eficaz contra parasitas da mesma espécieit, vem sendo descrita há longo tempo.

MOORE \& LANIER “ descreveram o aparecimento da resistência à cloroquina, em 1961, na Colômbia. Hoje, numerosas regiões apresentam o grave problema da resistência a esta 4 aminoquinoleína, incluindo o Sudeste Asiático ${ }^{14}$, Indochina, Malásia, Indonésia, $\mathrm{Pa}$ pua Nova Guiné15, Kênia, Tanzânia, Sudão, Zaire e mais recentemente Angolabe 10 .
O primeiro relato de falha no tratamento com o quinino data de 1910, no Brasil9. Apesar de ser o quinino, ainda hoje, a droga preconizada pela $\mathrm{OMS}^{13}$ para o tratamento de malária falcípara grave, não podemos ignorar que, apesar de raros, outros relatos de resistência foram descritos, como por exemplo na Tailândia ${ }^{7}{ }^{11}$.

A mefloquina (4-quinolinometanol) é considerada atualmente uma alternativa terapêutica apropriada para o tratamento de P. falciparum resistente aos demais antimaláricos. Esta droga apresenta certas vantagens, como o fato de poder ser administrada em dose única, isolada ou em associação com outros medicamentos (sulfadoxina-pirimetamina), proporcionando

(1) Auxilio financeiro do (NPq-Polonoroeste 700)349/85.

(2) Laboratório de Malária da Superintendência de Controle de Endemias (SULEN), Secretaria de Estado da Saúde do Estado de São Paulo. Rua Cardeal Arcoverde, 2878. CEP 05408 São Paulo, SP., Brasil. 


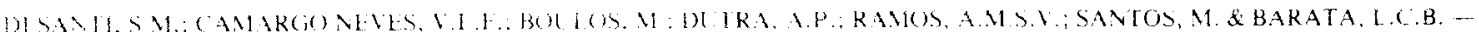

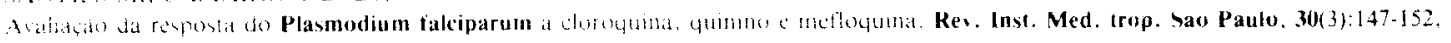
$198 \%$

uma rápila dimmulạa e negativaçào dos parasilai. Mas já existem relatos de resistência "in vi(0" na Tailândia e Leste da Áfrical" ?. No Brasil, estudos recentes tem demonstrado casos de cepas resistentes "in vitro" e "in vivo" a mefloquina isolada ou em tripla combinação : 13. Porém, por sua vida média ser bastante longa ( 21 dias), constitui-se de fator importante na seleção de cepas resistentes, visto que individuos com baixa dosagem da droga no sangue periférico, permanecendo em áreas com possibilidade de transmissão, estarào transferindo baixos niveis de mefloquina ao mosquito, facilitando o desenvolvimento de resistência. Com base nestes dados, torna-se imprescindivel a pesquisa de outras alternativas terapêuticas para o tratamento da malária causada por Plasmodium falciparum.

\section{MATERIAI. E MÉTODOS}

Os estudos foram realizados no periodo de novembro de 1984 a fevereiro de 1988. Após o diagnostico da espécie de plasmódio, realizado pelo Laboratorio de Malária da SUCEN, os pacientes se submeteram a punção venosa para relirada dia amostra de sangue, colhido em seringa heparinizada.

A parasitemia variou de 5.000 a 100.000 formas assexuadas por milímetro cúbico e foram triados para coleta, pacientes que não apresentassem relato de tratamento pregresso com drogas antimaláricas.

Os testes foram baseados na técnica de RILCKIIANN c cols. 1978'z

$O$ sangue parasitado foi lavado duas vezes com RPMI 1640 (Flow), suplementado com bicarbonato de sódio (Merck, $2 \mathrm{mg} / \mathrm{ml}$ ). Hepes (Signia, $6 \mathrm{mg} / \mathrm{ml}$ ) e sulfato de gentamicina (Schering. $40 \mu \mathrm{g} / \mathrm{ml}$ ). Desprezou-se a papa leucocitária. A seguir este sangue foi diluido para um hematócrito de 5\% em RPMI completo, isto 6 complementado com bicarbonato de sio dio. Hepes, sulfato de gentamicina e soro humano $\mathrm{AB}^{+}$ou $\mathrm{A}^{*}$.

As placas pré-doseadas contendo as várias concentraçōes da droga, em picomoles, foram fornecidas pela O.M.S. As drogas utilizadas foram a cloroquina, quinino e metlou uina. 10uadro! !

Gangue parasitado diluido foi distribuido r.s. placas de microtestes $(50 \mu \mathrm{l} / \mathrm{alveolo})$ e a segut incubado em dessecador com atmosfera de
50 de $\mathrm{CO}_{2}$, a $37^{\circ} \mathrm{C}$. Em rodos os restes foi feito um controle $(K)$ sem droga para verificaçào da maturação dos anéis até esquizontes. O tempo minimo de incubação foi de 24 horas e o máximo de 48 horas. Após este intervalo foram realizadas lâminas-controle (gota espessa corada por Giemsa a $2 \%$ em solução tamponada $\mathrm{pH}$ $7,02)$, para constatar a presença de esquizontes (parasitas com 3 ou mais núcleos). Foram considerados os testes contendo na lâmina-contrule número igual ou maior que $20 \%$ de esquizontes em 200 formas assexuadas.

QUADRO 1

Concentração das drogas por alvéolo

\begin{tabular}{llllrrrrr}
\hline $\begin{array}{l}\text { Dose (pmol) } \\
\text { Drogat }\end{array}$ & A & B & C & D & E & F & G & H \\
\hline Cloroquina & 0 & 1 & 2 & 4 & 5.7 & 8 & 16 & 32 \\
Quinino & 0 & 4 & 8 & 16 & 32 & 64 & 128 & 256 \\
Mefloquina & 0 & 0.5 & 1 & 2 & 4 & 5,7 & 8 & 16 \\
\hline
\end{tabular}

Foram classificadas como resistentes aquelas cepas que apresentaram esquizontes na presença de 5,7 ou mais picomoles de cloroquina, 64 ou mais picomoles de quinino e 4 ou mais picomoles de mefloquina, seguindo os critérios estabelecidos pela O.M.S.

Quanto à procedência das cepas de Plasmodium falciparum, $56,8 \%$ foram provenientes de pacientes que adquiriram a infecção no Estado de Rondônia, 16,9\% no Estado de Mato Grosso, $15,7 \%$ no Estado do Pará, 3,9\% no Estado do Acre, $2 \%$ no Estado de Goiás e 2\% de Estado indeterminado (Acre ou Rondônia).

\section{RESULTADOS}

Foram realizados 46 microtestes de sensibilidade com a cloroquina. Os resultados mostraram 46 cepas com crescimento dos parasitas até o estágio de esquizontes, em concentração igual ou superior a 5,7 picomoles/orificio, havendo, portanto, $100 \%$ de resistência "in vitro" à cloroquina.

Quanto ao quinino, dos 42 testes realizados, apenas 01 apresentou crescimento em 64 picomoles $(2,4 \%)$. sendo, portanto, considerado resistente.

Os resultados com a mefloquina mostraram $31,4 \%$ de resistência, 1 sto é, dos 51 micro- 
DI SANTI, S.M.; CAMARGO NEVES, V.L.F.; BOULOS, M.; DUTRA, A.P.; RAMOS, A.M.S.V.; SANTOS, M. \& BARATA, L.C.B. Avaliaçào da resposta do Plasmodium falciparum a cloroquina, quinino e mefloquiná. Rev. Inst. Med. trop. São Paulo, 30(3):147-152, 1988.

Lestes realizados, 16 apresentaram esquizontes em concentraçăo igual ou maior que 4 picomoles/orificio.

2.

Estes dados estão apresentados no Quadro

Com base nos testes realizados, segundo a procedência, não observamos áreas com maior incidência de resistência às drogas estudadas, havendo uma distribuição uniforme de cepas resistentes. Tabelas 1,2 e 3 .

\section{QUADRO 2}

Porcentagem de cepas sensíveis e resistentes à cloroquina, quinino e mefloquina

\begin{tabular}{lcc} 
& $\mathrm{S}$ & $\mathrm{R}$ \\
\hline $\begin{array}{l}\text { Cloroquina } \\
\mathrm{n}=46\end{array}$ & $0(0 \%)$ & $46(100 \%)$ \\
\hline $\begin{array}{l}\text { Quinino } \\
\mathrm{n}=42\end{array}$ & $41(97,6 \%)$ & $01(2,4 \%)$ \\
$\begin{array}{l}\text { Mefloquina } \\
\mathrm{n}=51\end{array}$ & $35(68,6 \%)$ & $16(31,4 \%)$ \\
\end{tabular}

No periodo de 1984 a 1986, houve um aumento significativo do índice de resistência à mefloquina. Não foi possivel avaliar se este quadro se mantém até hoje, devido ao pequeno número de testes realizados apos 1986. Na tabela 4 , observamos o padrão de resistência da mefloquina segundo o período de realização do teste.

\section{DISCUSSÃO}

O conhecimento do grau de sensibilidade do $\mathbf{P}$. falciparum aos diferentes medicamentos é de muito anseio de todos aqueles que deparam com as dificuldades de tratar um paciente acometido por tal protozoose.

A despeito de existirem dados sobre os microtestes de sensibilidade em nosso meio, estes são esparsos, não trazendo informaçôes uniformes e não correlacionando tais dados com a susceptibilidade "in vivo".

Os pacientes atendidos no Laboratório de Malária da SUCEN, são oriundos de várias regiões malarígenas do Brasil, sendo amostragem do que ocorre no país.

Os microtestes de sensibilidade, segundo a

TABELA 1

Resposta "in vitro" do P. falciparum à cloroquina, segundo a procedência

\begin{tabular}{|c|c|c|c|c|c|c|c|}
\hline \multirow[b]{2}{*}{ Estado } & \multicolumn{7}{|c|}{ Cepas com crescimento até dada concentração em pmoles/alvéolo } \\
\hline & 1 & 2 & 4 & 5,7 & 8 & 16 & 32 \\
\hline $\begin{array}{l}\text { Rondônia } \\
n=26\end{array}$ & $\begin{array}{l}26 \\
(100 \%)\end{array}$ & $\begin{array}{l}26 \\
(100 \%)\end{array}$ & $\begin{array}{l}26 \\
(100 \%)\end{array}$ & $\begin{array}{l}26 \\
(100 \%)\end{array}$ & $\begin{array}{l}22 \\
(85 \%)\end{array}$ & $\begin{array}{l}18 \\
(69 \%)\end{array}$ & $\begin{array}{l}08 \\
(31 \%)\end{array}$ \\
\hline $\begin{array}{l}\text { Mato Grosso } \\
n=09\end{array}$ & $\begin{array}{l}09 \\
(100 \%)\end{array}$ & $\begin{array}{l}09 \\
(100 \%)\end{array}$ & $\begin{array}{l}09 \\
(100 \%)\end{array}$ & $\begin{array}{l}09 \\
(100 \%)\end{array}$ & $\begin{array}{l}07 \\
(78 \%)\end{array}$ & $\begin{array}{l}07 \\
(78 \%)\end{array}$ & $\begin{array}{l}02 \\
(22 \%)\end{array}$ \\
\hline $\begin{array}{l}\text { Pará } \\
n=08\end{array}$ & $\begin{array}{l}08 \\
(100 \%)\end{array}$ & $\begin{array}{l}08 \\
(100 \%)\end{array}$ & $\begin{array}{l}08 \\
(100 \%)\end{array}$ & $\begin{array}{l}08 \\
(100 \%)\end{array}$ & $\begin{array}{l}07 \\
(87 \%)\end{array}$ & $\begin{array}{l}06 \\
(75 \%)\end{array}$ & $\begin{array}{l}04 \\
(50 \%)\end{array}$ \\
\hline $\begin{array}{l}\text { Acre } \\
n=01\end{array}$ & $\begin{array}{l}01 \\
(100 \%)\end{array}$ & $\begin{array}{l}01 \\
(100 \%)\end{array}$ & $\begin{array}{l}01 \\
(100 \%)\end{array}$ & $\begin{array}{l}01 \\
(100 \%)\end{array}$ & $\begin{array}{l}0 i \\
(100 \%)\end{array}$ & $\begin{array}{l}01 \\
(100 \%)\end{array}$ & $\begin{array}{l}01 \\
(100 \%)\end{array}$ \\
\hline $\begin{array}{l}\text { Goíás } \\
\mathrm{n}=01\end{array}$ & $\begin{array}{l}01 \\
(100 \%)\end{array}$ & $\begin{array}{l}01 \\
(100 \%)\end{array}$ & $\begin{array}{l}01 \\
(100 \%)\end{array}$ & $\begin{array}{l}01 \\
(100 \%)\end{array}$ & $\begin{array}{l}01 \\
(100 \%)\end{array}$ & $\begin{array}{l}01 \\
(100 \%)\end{array}$ & $\begin{array}{l}01 \\
(0 \%)\end{array}$ \\
\hline $\begin{array}{l}\text { Indeterminado } \\
\mathbf{n}=01\end{array}$ & $\begin{array}{l}01 \\
(100 \%)\end{array}$ & $\begin{array}{l}01 \\
(100 \%)\end{array}$ & $\begin{array}{l}01 \\
(100 \%)\end{array}$ & $\begin{array}{l}01 \\
(100 \%)\end{array}$ & $\begin{array}{l}01 \\
(100 \%)\end{array}$ & $\begin{array}{l}01 \\
(100 \%)\end{array}$ & $\begin{array}{l}01 \\
(0 \%)\end{array}$ \\
\hline
\end{tabular}


1)I SANII, S.M.; CAMARGO NEVES, V.L.F.; BOULOS, M.; DUTRA, A.P.; RAMOS, A.M.S.V.; SANTOS, M.\&\& BARATA, L.C.B. Avalią̧ão da resposıa do Plasmodium falciparum a cloroquina, quinino e mefloquina. Rev. Inst. Med. trop. Sāo Paulo, 30(3): 147-152, 1988 .

lécnica de Rieckmann, mostraram ausência de sensibilidade à cloroquina em todas as $\mathbf{4 6}$ amostras testadas.

Apesar da reconhecida resistência do $\mathbf{P}$. falciparum à cloroquina, esta droga continua a ser empregada em vários locais, dentre os quais o Brasil, mostrando ser ainda útil, pelo menos para evitar mortalidade 4 .

TABELA 2

Resposta "in vitro" do P. falciparum ao quinino, segundo a procedência

\begin{tabular}{|c|c|c|c|c|c|c|c|}
\hline \multirow[b]{2}{*}{ Estado } & \multicolumn{7}{|c|}{ Cepas com crescimento até dada concentração em pmoles/alvéolo } \\
\hline & 4 & 8 & 16 & 32 & 64 & 128 & 256 \\
\hline $\begin{array}{l}\text { Rondônia } \\
n=24\end{array}$ & $\begin{array}{l}24 \\
(100 \%)\end{array}$ & $\begin{array}{l}24 \\
(100 \%)\end{array}$ & $\begin{array}{l}13 \\
(54 \%)\end{array}$ & $\begin{array}{l}07 \\
(29 \%)\end{array}$ & $\begin{array}{l}0 \\
(0 \%)\end{array}$ & $\begin{array}{l}0 \\
(0 \%)\end{array}$ & $\begin{array}{l}0 \\
(0 \%)\end{array}$ \\
\hline $\begin{array}{l}\text { Mato Grosso } \\
\mathrm{n}=08\end{array}$ & $\begin{array}{l}08 \\
(100 \%)\end{array}$ & $\begin{array}{l}08 \\
(100 \%)\end{array}$ & $\begin{array}{l}06 \\
(75 \%)\end{array}$ & $\begin{array}{l}03 \\
(37 \%)\end{array}$ & $\begin{array}{l}0 \\
(0 \%)\end{array}$ & $\begin{array}{l}0 \\
(0 \%)\end{array}$ & $\begin{array}{l}0 \\
(0 \%)\end{array}$ \\
\hline $\begin{array}{l}\text { Pará } \\
\mathrm{n}=07\end{array}$ & $\begin{array}{l}07 \\
(100 \%)\end{array}$ & $\begin{array}{l}07 \\
(100 \%)\end{array}$ & $\begin{array}{l}04 \\
(57 \%)\end{array}$ & $\begin{array}{l}02 \\
(29 \%)\end{array}$ & $\begin{array}{l}0 \\
(0 \%)\end{array}$ & $\begin{array}{l}0 \\
(0 \%)\end{array}$ & $\begin{array}{l}0 \\
(0 \%)\end{array}$ \\
\hline $\begin{array}{l}\text { Acre } \\
n=01\end{array}$ & $\begin{array}{l}01 \\
(100 \%)\end{array}$ & $\begin{array}{l}01 \\
(100 \%)\end{array}$ & $\begin{array}{l}0 \\
(0 \%)\end{array}$ & $\begin{array}{l}0 \\
(0 \%)\end{array}$ & $\begin{array}{l}0 \\
(0 \%)\end{array}$ & $\begin{array}{l}0 \\
(0 \%)\end{array}$ & $\begin{array}{l}0 \\
(0 \%)\end{array}$ \\
\hline $\begin{array}{l}\text { Goiás } \\
n=01\end{array}$ & $\begin{array}{l}01 \\
(100 \%)\end{array}$ & $\begin{array}{l}01 \\
(100 \%)\end{array}$ & $\begin{array}{l}01 \\
(100 \%)\end{array}$ & $\begin{array}{l}01 \\
(100 \%)\end{array}$ & $\begin{array}{l}01 \\
(100 \%)\end{array}$ & $\begin{array}{l}0 \\
(0 \%)\end{array}$ & $\begin{array}{l}0 \\
(0 \%)\end{array}$ \\
\hline $\begin{array}{l}\text { Indeterminado } \\
n=01\end{array}$ & $\begin{array}{l}01 \\
(100 \%)\end{array}$ & $\begin{array}{l}01 \\
(100 \%)\end{array}$ & $\begin{array}{l}0 \\
(0 \%)\end{array}$ & $\begin{array}{l}0 \\
(0 \%)\end{array}$ & $\begin{array}{l}0 \\
(0 \%)\end{array}$ & $\begin{array}{l}0 \\
(0 \%)\end{array}$ & $\begin{array}{l}0 \\
(0 \%)\end{array}$ \\
\hline
\end{tabular}

TABELA 3

Resposta "in vitro" do P. falciparum à mefloquina, segundo a procedência

\begin{tabular}{|c|c|c|c|c|c|c|c|}
\hline \multirow[b]{2}{*}{ Estado } & \multicolumn{7}{|c|}{ Cepas com crescimento até dada concentração em pmoles/alvéolo } \\
\hline & 0,5 & 1 & 2 & 4 & 5,7 & 8 & 16 \\
\hline $\begin{array}{l}\text { Rondônia } \\
\mathrm{n}=29\end{array}$ & $\begin{array}{l}29 \\
(100 \%)\end{array}$ & $\begin{array}{l}29 \\
(100 \%)\end{array}$ & $\begin{array}{l}25 \\
(86 \%)\end{array}$ & $\begin{array}{l}08 \\
(27 \%)\end{array}$ & $\begin{array}{l}05 \\
(17 \%)\end{array}$ & $\begin{array}{l}01 \\
(03 \%)\end{array}$ & $\begin{array}{l}0 \\
(0 \%)\end{array}$ \\
\hline $\begin{array}{l}\text { Mato Grosso } \\
n=10\end{array}$ & $\begin{array}{l}10 \\
(10 \%)\end{array}$ & $\begin{array}{l}10 \\
(100 \%)\end{array}$ & $\begin{array}{l}08 \\
(80 \%)\end{array}$ & $\begin{array}{l}03 \\
(30 \%)\end{array}$ & $\begin{array}{l}0 \\
(0 \%)\end{array}$ & $\begin{array}{l}0 \\
(0 \%)\end{array}$ & $\begin{array}{l}0 \\
(0 \%)\end{array}$ \\
\hline $\begin{array}{l}\text { Pará } \\
\mathrm{n}=08\end{array}$ & $\begin{array}{l}08 \\
(100 \%)\end{array}$ & $\begin{array}{l}08 \\
(100 \%)\end{array}$ & $\begin{array}{l}07 \\
(87 \%)\end{array}$ & $\begin{array}{l}03 \\
(37 \%)\end{array}$ & $\begin{array}{l}0 \\
(0 \%)\end{array}$ & $\begin{array}{l}0 \\
(0 \%)\end{array}$ & $\begin{array}{l}0 \\
(0 \%)\end{array}$ \\
\hline $\begin{array}{l}\text { Acre } \\
\mathrm{n}=2\end{array}$ & $\begin{array}{l}02 \\
(100 \%)\end{array}$ & $\begin{array}{l}02 \\
(100 \%)\end{array}$ & $\begin{array}{l}01 \\
(50 \%)\end{array}$ & $\begin{array}{l}0 \\
(0 \%)\end{array}$ & $\begin{array}{l}0 \\
(0 \%)\end{array}$ & $\begin{array}{l}0 \\
(0 \%)\end{array}$ & $\begin{array}{l}0 \\
(0 \%)\end{array}$ \\
\hline $\begin{array}{l}\text { Goiás } \\
n=1\end{array}$ & $\begin{array}{l}01 \\
(100 \%)\end{array}$ & $\begin{array}{l}01 \\
(100 \%)\end{array}$ & $\begin{array}{l}01 \\
(100 \%)\end{array}$ & $\begin{array}{l}01 \\
(100 \%)\end{array}$ & $\begin{array}{l}01 \\
(100 \%)\end{array}$ & $\begin{array}{l}0 \\
(0 \%)\end{array}$ & $\begin{array}{l}0 \\
(0 \%)\end{array}$ \\
\hline $\begin{array}{l}\text { Indeterminado } \\
\mathrm{n}=1\end{array}$ & $\begin{array}{l}01 \\
(100 \%)\end{array}$ & $\begin{array}{l}01 \\
(100 \%)\end{array}$ & $\begin{array}{l}01 \\
(100 \%)\end{array}$ & $\begin{array}{l}01 \\
(100 \%)\end{array}$ & $\begin{array}{l}0 \\
(0 \%)\end{array}$ & $\begin{array}{l}0 \\
(0 \%)\end{array}$ & $\begin{array}{l}0 \\
(0 \%)\end{array}$ \\
\hline
\end{tabular}


DI SANTI, S.M.; CAMARGO NEVES, V.L.F.; BOULOS, M.; DUTRA. A.P.; RAMOS, A.M.S.V.: SANTOS, M. \& BARATA, L.C.B. Avaliação da resposta do Plasmodium falciparum a cloroquina, quinino e melloquina. Rev. Inst. Med. trop. São Paulo, 30(3): 147-152, 1988.

Nos testes realizados em 42 amostras para o quinino, observou-se apenas 1 resistente. Este padrão de resposta confronta com os dados obtidos por KNOWLEs e col, que enconraran paralelismo de resposta entre a cloroquina e o quinino nos testes "in vitro".

No entanto, em 7 pacientes que tivemos a oportunidade de utilizar o sulfato de quinino na dosagem de $25 \mathrm{mg} / \mathrm{kg} /$ dia durante 7 dias, e de cujas amostras foram realizados microtestes de sensibilidade, observamos ausência de correlação. Enquanto todos os testes "in vitro" mostraram sensibilidade do $\mathbf{P}$. falciparum ao quinino, 3 pacientes recrudesceram com tratamento completo.

Tal fato demonstra a nevessidade de se reestudar o limite de resistencia, nara os testes "in vitro", relativos ao quinino. na malária brasileira.

Com a mefloquina um fato surpreendente chama a atenção. Apesar desta droga não ter sido rotineiramente empregada durante a epoca do estudo, observou-se baixa sensibilidade "in vitro" do P. falciparum a ela, sensibilidade essa que foi decrescendo com o desenvolver do esiudo (tabela 4).

Um outro fato que merece destaque é que, na mesma época, estudos em humanos, mostram resistência bem menor da observada "in vitro"'?.

A exemplo do quinino, aqui também se faz necessário reavaliação do limiar de resistência nos testes "in vitro", para a malária adquirida no Brasil.

A despeito da variada procedencia dos casos estudados nesse trabalho, julgamos necessário estudos mais abrangentes, procurando

TABELA 4

Distribuição por ano, dos níveis de resposta à mefloquinà

\begin{tabular}{llllll}
\hline Dose $^{*}$ & 1984 & 1985 & 1986 & 1987 & 1988 \\
\hline 0,5 & 0 & 0 & 0 & 0 & 0 \\
1 & 01 & 06 & 0 & & 01 \\
2 & 04 & 18 & 01 & $\ldots$ & 04 \\
$4 * *$ & 0 & 08 & 02 & - & 0 \\
5,7 & 0 & 03 & 02 & -- & 0 \\
8 & 0 & 01 & 0 & & 0 \\
16 & 0 & 0 & 0 & $-\ldots$ & 0 \\
\hline TOTAL & 05 & 36 & 05 & 0 & 05 \\
\hline * picomoles & & & & \\
** limiar de sensibilidade & &
\end{tabular}

comparar microtestes de sensibilidade com evolução clínica dos pacientes, possibilitando assim, que amostragem maior esclareça algumas dúvidas aqui abordadas.

\section{SUMMARY}

\section{Evaluation of Plasmodium falciparum response to chloroquine, quinine and mefloquine}

The present study is concerned with the analysis of Plasmodium falciparum strains from the Brazilian Amazon Region, collected at the Malaria Laboratory - SUCEN". "In vitro" sensitivity tests were performed using chloroquine (46 samples), quinine (42 samples) and inefloquine (51 samples). Results have shown "in vitro" resistance to chloroquine in $100 \%$ of the tested samples. to quinine in $2.4 \%$ and to mefloquine in $31.4 \%$. Seven patients were treated with quinine and nine with the triple combination (mefloquine plus sulfadoxine plus pyrimethamine). No correlation was shown between the therapeutic response and the "in vitro" tests.

\section{REFERÊNCIAS BIBLIOGRÁFICAS}

1. BOUDREAU, E.F. - Type II mefloquine resistance in Thailand. Lancet, 2: 1335, 1982.

2. BOULOS, M.; DI SANTI, S.M.; BARATA, L.C.B.; SEGURADO, A.A.C.; DUTRA, A.P. \& CAMARGO NEVES, V.L.F. - Some aspects of treatment, prophylaxis and chemoresistance of Plasmodium falciparum malaria. Mem. Inst. Oswaldo Cruz, 81 (Suppl. 2): 255-257, 1986

3. BYGJBERG, I.C.; SCHAPIRA, A.; FLACHS, H.; GOMME, G. \& JEPSEN, S. - Mefloquine resistance of falciparum malaria from Tanzania enhanced by treatment. Lancet, 1: 774-775, 1983.

4. HOIIMAN, S.L.; MASBAR, S.; HUSSEN, P.R.; SOEWUSTA, A.; HARVIN, S.; MASWOTO, H.A.; CAMPBELL, J.R.; SMAKOVSKI, L. \& WINDY, I. - Abseince of malaria mortality in villagers with chloroquine-resistant Plasmodium falciparum treated with chloroquine. Trans. roy. Soc. trop. Med. Hyg., 78: $175-178,1984$.

5. KNOWIIS, G.: DAVIDSON, W. 2, LOLLEY, D. \& AIPRES, M.P. - The relationship between the "in vitro" response of Plasmodium falciparum 10 chloroquine, quinine and melloquine. Trans. roy. Soc. trop. Med. Hyg., 78: 146-150, 1984

6. KYRONSEPPA, H.; LUMIO, i.; UKKONEN, R. \& PETTERSON, T. - Chloroquine-resistant malaria from Angola. Lancet, 1: 1244, 1984.

7. MIGASENA, S.; BUNNAG, D. \& HARINASUTA, T. 
DISANTI, S.M.; CAMARGO VEVES, V.L.F.: BOUIOS. N.; DLTRA. A.P.; RAMOS, A.M.S.V.; SANTOS, M. \& BARAIA.I C.B. Avaliaçăo da resposta do Plasmodium falciparum à cloroquina, quitino e mefloquina. Rev, Inst. Med. trop. São Paulo. 301:14:147-152. 1988.

- A case of Plasmodium falciparum in Thailand apparently resistant to quinine. Ann. trop. Med. Paravit., 74: 24,3-244, 1980 .

8. MOORE, D.V. \& LANIER, I.E. - Obicrvations on iwo Plasmodium falciparum with an abnornal response to chloroquine. Amer. J. trop. Med. Hyg., 10: $5-9,1961$

9. NOCHT, B. \& WENER, H. - Beobachtungen ube relative Chininresitenz bei Malaria aus Brasilien. Dtsch. med. Wschr., 36: 1557-1560, 1910.

10. OLSEN, V.V.; JENSFN, T. \& JORGENSEN, M. Chloroquine-resistant Plasmodium falciparum malaria from Angola. Lancet, 1: 1462-1463, 1984

11. REACHER, M.; CAMPBELL, C.C.; FREEMAN, J.; DOBERSTYN, E.B. \& BRANDLING-BENNETT, A.D. - Drug therapy for Plasmodium falciparum malaria resistant to pyrimethamine-sulfadoxine (Fansidar). A study of alternate regimens in Lastern Thailand, 1980. Lancet, 2: 1066-1069, 1981

12. RIECKMANN, K.H.; SAX, L.J.; CAMPBELL, G.H.
\& MREMA, J.E. - Drug sensitivity of Plasmodium falciparum. An "in vitro" microtechnique. lancet, I:22-23, 1978

13. SOUZA, J.M. de; SHETH, U.K.: OLIVEIRA. R.M.G.; GOMES, A.T. \& CAVAI CANTE, E.Q. A phase I clinical trial of Fansimef (Mefloquine plus sulfadoxine-pyrimethamine) in Brazilian male subjects. Bull. WId. HIth. Org., 63: 611-615, 1985.

14. UPRETY, H.C.; GUPTA, V.K.\& SHARMA, V.P. Modified plan of operation and its impact on malaria. Indian J. Malar., 19:137-138, 1982.

15. WERNSDORFER, W. - Known geographical distribution, frequency and intensity of drug-resistant malaria. (Cyclostyled report) $\mathrm{MAP} / \mathrm{SC}(\mathrm{M} / \mathrm{WP}$ 83.10. Geneva, World Health Organization, 1983.

16. WHO - Terminology of malaria and of malaria eradication. Report of a Dratting Committee. Geneva, World Health Organization, 1963. p. 116.

17. WHO - Advances in malaria chemotheraphy. Wld. HIth, Org. techn. Rep. Ser., (711). 1984. 